\title{
Pediatric unilateral knee swelling: a case report of a complicated differential diagnosis and often overlooked cause
}

https://doi.org/10.1515/jom-2020-0332

Received December 30, 2020; accepted August 25, 2021;

published online January 6, 2022

\begin{abstract}
Lyme disease is the most common vector-borne illness in the United States. However, Lyme arthritis is a diagnosis that is often missed, even in children, who are the population with the highest incidence of Lyme disease. Lyme arthritis, which presents in the later stage of Borrelia burgdorferi infection, needs to be recognized and managed promptly, especially in endemic areas or when exposure to ticks is known. We present a case of a 3-year-old female presenting to the emergency department with a history of limping for 2 weeks. The mother of the child recognized a tick bite. However, the child was not taken to seek care expeditiously, because she had not developed any rashes. Test results demonstrated that the patient was IgG positive and IgM negative for Lyme disease, with Western blot confirming the diagnosis of Lyme arthritis. Most patients presenting with Lyme arthritis do not recall having a tick bite, making it difficult to differentiate it from other pediatric conditions. When this diagnosis is missed, it can result in long-term morbidity, which is generally refractory to intravenous antibiotic therapy, oftentimes requiring synovectomy. Hence, this underscores the importance of the consideration of Lyme arthritis as a differential diagnosis in patients presenting with joint effusion.
\end{abstract}

Keywords: knee swelling; Lyme arthritis; Lyme disease.

In the United States, Lyme disease is mainly caused by Borrelia burgdorferi, an agent transmitted by Ixodes scapularis and Ixodes pacificus [1, 2]. It is estimated that in the United States, 476,000 cases of Lyme disease occurred

*Corresponding author: Katherine E. Guardado, OMS II, Michigan State University College of Osteopathic Medicine, 965 Wilson Rd, East Lansing, MI 48824, USA, E-mail: guardad6@msu.edu

Shane Sergent, DO, Institute for Global Health, Michigan State University College of Osteopathic Medicine, Lansing, MI, USA; Conemaugh Health System, Johnstown, PA, USA; and Michigan State University College of Osteopathic Medicine, Lansing, MI, USA annually between 2010 and 2018 [3]. Incidence in endemic areas is highest among children 5-15 years old and adults 45-55 years old [2]. Lyme disease is the most common vector-borne disease in the United States [4]. The majority of cases occur in the northeast (Maine to Maryland), the Midwest (Wisconsin and Minnesota), and some western states (northern California, Oregon, Nevada) [4]. In these areas, Lyme disease may be overdiagnosed leading to overtreatment, whereas in nonendemic areas, Lyme disease manifestations may be underdiagnosed $[5,6]$. In endemic areas, the overdiagnosis of Lyme arthritis has become a large issue, whereas in nonendemic areas, the diagnosis is often missed in children with acute arthritis [6]. Symptoms may vary and could be challenging to recognize considering that some symptoms are similar to those of other illnesses that present in childhood such as juvenile idiopathic arthritis [4, 7]. Hence, the issue lies in the fact that Lyme arthritis may not be considered as part of the initial differential in children with joint swelling [6].

Lyme disease may be asymptomatic or present in three stages: early localized, early disseminated, and late disease [8]. The early localized stage may comprise fevers, chills, headache, fatigue, muscle and joint pain, and erythema migrans (EM), which usually present 3-30 days after the tick bite [8]. The early disseminated stage presents after days or weeks of initial symptoms. It presents with fever, fatigue, headache, myalgia, or arthralgia. Severe symptoms include cranial nerve palsies, peripheral neuropathy, meningitis, and carditis. The most common nervous system involvement, particularly in children, is facial nerve palsy [8]. In the late stage, the most common presentation is intermittent or persistent arthritis in one or more large joints [8]. It is estimated that $50-60 \%$ of all patients with untreated Lyme disease will develop Lyme arthritis [9]. Children have a greater probability of developing arthritis due to $B$. burgdorferi compared to adults and are more likely to have Lyme arthritis as the only symptom [9]. Most patients present with monoarthritis of the knee, which is arthralgia followed by arthritis or the sudden start of a swollen joint [2]. Lyme arthritis encompasses recurrent rounds of joint swelling and pain that 


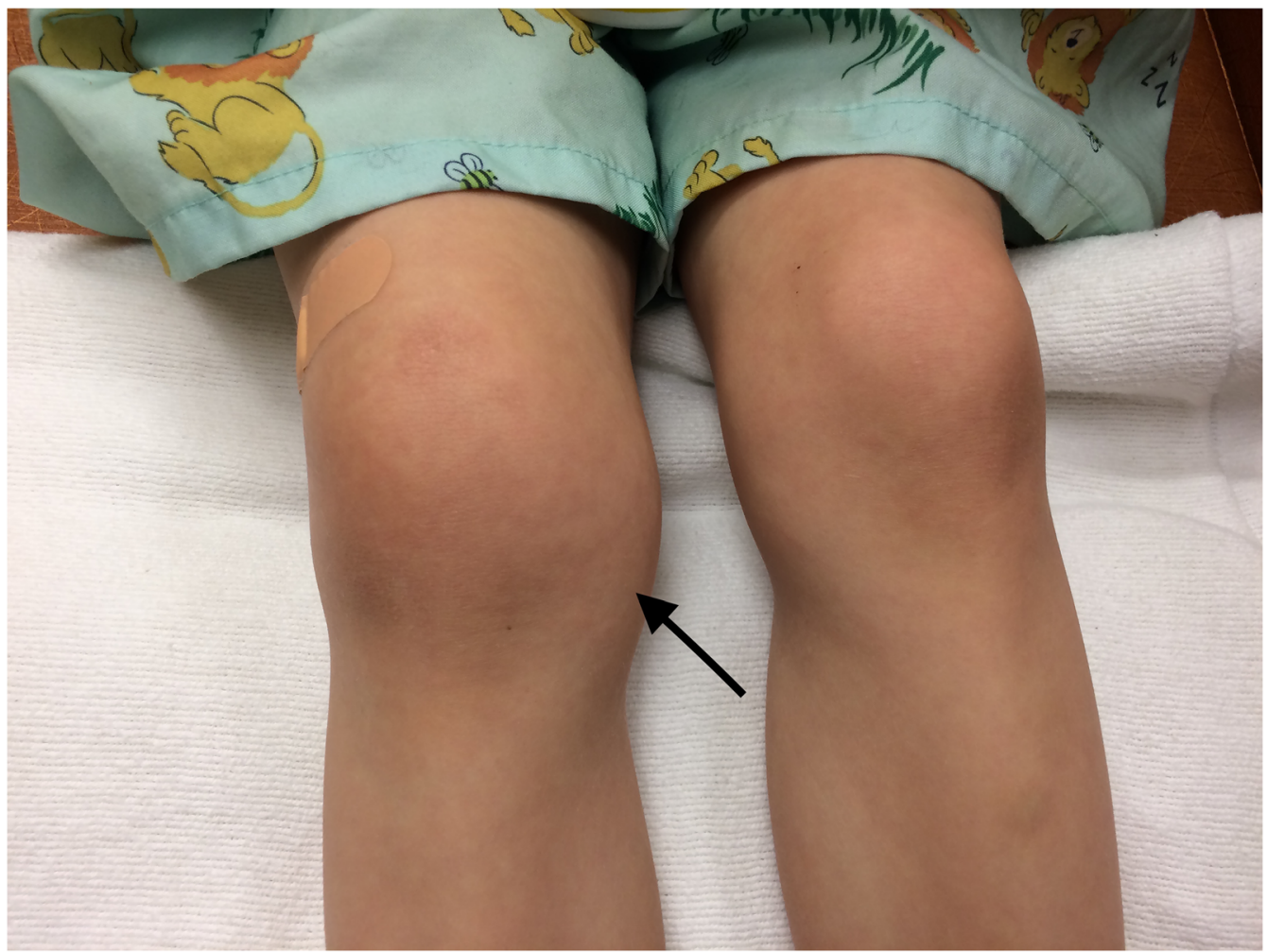

Figure 1: Anterior view of the legs demonstrates right knee swelling.

stop and start at irregular intervals that could last for days or months [1]. In children, it mostly affects the knee; however, multiple joints may also be affected such as the shoulder, elbow, ankle, and temporomandibular joint $[1,6]$.

A retrospective study looking at the medical records of children diagnosed with Lyme arthritis at a Children's Hospital in Western New York found that Lyme arthritis may present with symptoms akin to other forms of arthritis, such as oligoarticular juvenile idiopathic arthritis [7]. This could potentially lead to the misdiagnosis of Lyme arthritis [7]. Hence, it is essential for physicians to keep in mind that testing is necessary for making the diagnosis of juvenile idiopathic arthritis over Lyme arthritis [7]. Increasing the awareness of Lyme disease and Lyme arthritis in nonendemic areas could lead to an earlier diagnosis and better management of the disease [7]. Lyme arthritis is usually seen in primary care, rheumatology, and orthopedic settings [1]. Given the high risk of joint damage and systemic manifestations, prompt recognition and treatment is necessary [1]. Lyme arthritis should be included as part of the differential diagnosis, even without recognition of a tick bite, because most children who develop Lyme disease are not aware that they have it [10]. It could take days to obtain results for serology testing, and treatment must be started even before diagnostic results are available [11].

\section{Case description}

In December 2016, a 3-year-old female presented to a hospital in Pennsylvania with a history of painless swelling and limping for 2 weeks, preferring her left leg for ambulation. The mother stated that the child had a history of right knee swelling (Figure 1) that became worse in the evening, so she promptly brought the child into the emergency department for evaluation. The mother denied trauma, fever, night sweats, weight loss, anorexia, or meningeal symptoms. The child had no history of developmental delays or similar symptoms, as well as no family history, bleeding disorders, medications, or preceding viral symptoms. The mother denied any history of pain, insect bites, change in activity, bowel or bladder changes, and motor or neurological changes. The mother reported that her child's knee seemed to make the child uncomfortable with movement and denied any other modifying or associated systemic symptoms. The mother initially denied history of a tick bite, yet after focused questioning, the mother stated that the child had ticks on her approximately 9 months ago. However, because the child was checked daily and had no history of skin rashes, she was not promptly taken to see a physician. She also had no history suggestive of early localized or early disseminated Lyme disease. 
Table 1: Results of hematological examination.

\begin{tabular}{lrr}
\hline Parameter & Admission value & Reference range \\
\hline WBC count, $10 * 3 / \mu \mathrm{L}$ & 11.5 & $6.0-17.5$ \\
RBC count, $10 \star 3 / \mu \mathrm{L}$ & 3.9 & $4.10-5.30$ \\
Hemoglobin, gm/dL & 11.3 & $11.3-14.1$ \\
Hematocrit, \% & 34.0 & $33-41$ \\
Neutrophils, \% & 57.0 & $20-40$ \\
Lymphocytes, \% & 31.0 & $55-67$ \\
Monocytes, \% & 9.0 & $0-10$ \\
Eosinophils, \% & 3.0 & $0-6$ \\
Basophils, \% & 1.0 & $0-2$ \\
Platelets, $10 * 3 \mu \mathrm{L}$ & 139,000 & $130,000-400,000$ \\
\hline
\end{tabular}

WBC, white blood cell; RBC, red blood cell.

Table 2: Comprehensive metabolic panel.

\begin{tabular}{lrr}
\hline Parameter & Admission value & Reference range \\
\hline Sodium, mmol/L & $137(\mathrm{~L})$ & $138-145$ \\
Chloride, mmol/L & 106 & $98-107$ \\
$\mathrm{CO}_{2}, \mathrm{mmol} / \mathrm{L}$ & 21 & $20-28$ \\
Anion gap, mmol/L & 10 & 44,330 \\
Calcium, mg/dL & 9.6 & $8.8-10.8$ \\
BUN, mg/dL & 9 & 44,333 \\
Creatinine, mg/dL & 0.5 & $0.5-1.0$ \\
Albumin, g/dL & 4.3 & $3.8-5.4$ \\
Total bilirubin, mg/dL & 0.1 & $0.2-1.2$ \\
Alkaline phosphatase, U/L & 204 & $0-500$ \\
Total protein, g/dL & 7.1 & $6.0-8.0$ \\
AST, IU/L & 31 & $5-34$ \\
ALT, IU/L & 13 & $0-55$ \\
Glucose, fasting, mg/dL & 113 & $60-100 \mathrm{mg} / \mathrm{dL}$ \\
\hline
\end{tabular}

AST, aspartate aminotransferase; ALT, alanine aminotransferase; BUN, blood urea nitrogen.

On physical examination, the patient was in no distress. She was afebrile with normal vitals. The child demonstrated right knee effusion with signs of crepitus and decreased range of motion secondary to swelling. Motor and neurological exams were normal. The right knee landmarks were obscured due to effusion. The anterior drawer test and McMurray test were negative. In addition, she had an unsteady gait without joint tenderness, crepitus, or overlying skin pathology. The osteopathic structural exam showed no valgus or varus instability. There were no other positive findings on examination.

Serology for B. Burgdorferi antigen was ordered. Western blot confirmed the presence of IgG antibodies (positive for p93, p66, p58, p41, p39, p28, p23, and p18 bands). IgM antibodies were negative because only band $\mathrm{p} 41$ came back positive. Comprehensive metabolic panel and complete blood count results were all in the normative range, except for a white blood cell count of $11.5 \times 10^{9}$ cells $/ \mu \mathrm{L}$ and neutrophils count of $57 \%$ (Tables 1 and 2). A joint arthrocentesis was performed in the ED that demonstrated a cloudy synovial fluid with 21,725 /cumulative white blood cells, 4,750/cumulative red blood cells, and a neutrophil count of $70 \%$. In addition, human leukocyte antigens-B27, antinuclear antibodies (ANA), cyclic citrullinated peptide (CCP), and rheumatoid factor (RF) were negative. Synovial fluid culture was negative, and Gram stain contained polymorphonuclear leukocytes. Magnetic resonance imaging demonstrated large right suprapatellar joint effusion with enlarged lymph nodes in the right popliteal fossa. The child received 2 days of intravenous Rocephin (ceftriaxone sodium) followed by a 21 day course of amoxicillin. She had complete resolution of symptoms within a few days of treatment and no return of symptoms 1 month following treatment resolution.

\section{Discussion}

Even though the current patient's mother eventually recalled the occurrence of tick bites, most patients presenting with Lyme arthritis do not recall having a tick bite, thus making it difficult to differentiate Lyme arthritis from other pediatric conditions [9]. For instance, septic arthritis, juvenile idiopathic arthritis, and transient synovitis also present with an elevated leukocyte count in synovial fluid and elevated acute reactants $[9,12]$. Lyme arthritis in children may present with synovial white blood cell count at similar levels observed in septic arthritis [9]. Untreated patients may develop early disseminated disease including migratory polyarthralgia with or without cardiac and neurologic symptoms [1]. Subsequently, without antibiotic treatment, Lyme arthritis could develop with attacks that last days, weeks, or months [2]. In addition, a delayed diagnosis may lead to the surgeon recommending joint irrigation and debridement for possible septic arthritis [13].

Diagnosis of Lyme arthritis is done through two-tier testing $[1,14,15]$. The first test involves a highly sensitive enzyme immunoassay (EIA) or immunofluorescence assay (IFA), and then a Western blot is performed to confirm the presence of antibodies [1]. However, this type of testing is not sensitive during early infection due to some patients being seronegative [14]. The CDC recommends the two-tier approach to be utilized in suspected disseminated Lyme disease [15]. If symptoms are present for less than 1 month, two or more IgM bands of 23, 39, or $41 \mathrm{kD}$ are needed for diagnosis [1]. IgM on Western blot has elevated rates of false positives, therefore it should not be utilized alone for diagnosis of patients experiencing symptoms for over a month [1]. A minimum of five of $10 \mathrm{IgG}$ bands of $18,23,28,30,39,41,45,58,66$, or $93 \mathrm{kD}$ 
are required for diagnosis during late infection [1]. Lyme disease has a strong IgG response, indicating antigens targeting the spirochete $B$. burgdorferi [1]. It persistently has positive serologies in EIA/IFA and C6 peptide enzyme-linked immunosorbent assay (ELISA), reflecting immune activation [1].

The patient highlighted in this case report was Lyme IgG positive and IgM negative. IgM antibodies are more transient and negative after 2-3 weeks from presentation of skin lesions, whereas IgG antibodies are detected after 4 weeks [9]. Because Lyme arthritis has a late disease manifestation, positive IgG antibodies to $B$. burgdorferi are present in our patient. This is in accordance with the patient's mother noticing ticks on the child months prior. When EM is present in the absence of laboratory results, EM is sufficient to treat Lyme disease [6]. In the present case, a joint arthrocentesis was performed to provide adjunct evidence for Lyme disease diagnosis. However, synovial fluid analysis is usually performed for diagnosis to rule out other causes of joint swelling such as staphylococcal septic arthritis [14]. In Lyme disease, other tests, such as rheumatoid factor or antinuclear antibodies, are usually negative or low titer may be present [14]. Inflammatory markers such as erythrocyte sedimentation rate (ESR) and c-reactive protein (CRP) may be elevated, but normal results do not exclude diagnosis [7]. In cases where ESR and CRP are elevated, there is an average ESR of $39-44.6 \mathrm{~mm} / \mathrm{h}$ and CRP of $2.7-38.5 \mathrm{mg} / \mathrm{L}$ [7]. Imaging studies such as MRI show nonspecific joint effusions, whereas MRI with contrast dye may show synovial thickening or enhancement [14]. MRI may be useful to visualize the presence of joint damage and aid in the planning of synovectomies by displaying the degree of synovitis within the joint [14].

When managing a patient with Lyme disease, different approaches are required depending on the stage of the disease. Early disease, which presents with EM, may be resolved without antibiotics [2]. However, treatment may reduce the duration of the disease and prevent the development of later stages [2]. For adults with EM, $100 \mathrm{mg}$ oral doxycycline administered twice a day for 10-21 days or 250-500 mg oral amoxicillin given three times a day for 10-21 days is recommended [2]. Cefuroxime axetil may be given when patients cannot be treated with doxycycline or amoxicillin [2]. Children with permanent dentition with EM can be treated with $1-2 \mathrm{mg} / \mathrm{kg}$ oral doxycycline twice a day or $25-50 \mathrm{mg} / \mathrm{kg}$ oral amoxicillin three times a day [2]. For children younger than 8 years old, amoxicillin is an effective therapy [2]. The CDC's recommendations for Lyme arthritis in adults include doxycycline (100 mg, twice per day orally), amoxicillin (500 mg, three times per day orally), and cefuroxime (500 mg, twice per day orally) for 28 days [16].
The recommendations for children older than 8 years old include doxycycline $(4.4 \mathrm{mg} / \mathrm{kg}$ per day orally, divided into two doses), amoxicillin (50 mg/kg per day orally, divided into three doses), and cefuroxime (30 mg/kg per day orally, divided into two doses) for 28 days [16]. Recommendations for children less than 8 years old are amoxicillin $(30 \mathrm{mg} / \mathrm{kg}$ per day orally, divided into two doses) [16]. It may also be beneficial to add anti-inflammatory medication and physical therapy [2]. Antibiotic therapy has good outcomes; therefore, surgical drainage should not be done until a Lyme arthritis diagnosis can be ruled out [12, 13]. In addition, other treatments may include osteopathic manipulative treatment (OMT). Although there are no current cases reporting the use of OMT for Lyme arthritis, OMT has been utilized to treat Lyme disease - induced Bell's palsy by targeting cranial, cervical, and sacral somatic dysfunction [17].

Lyme disease in children has a good prognosis, and most children have no long-term effects [12]. Even in the presence of Lyme arthritis, children may recover faster and even utilize fewer antibiotics compared to adults [9, 12]. Prevention measures include education, use of protective clothing, and repellent, especially in endemic areas [2]. A Lyme disease vaccine has been evaluated; however, the optimal intervention has not been established [2].

\section{Conclusions}

This case demonstrates the importance of including Lyme arthritis as part of the differential diagnosis of joint swelling regardless of the recollection of a tick bite [2]. Late findings of Lyme disease can occur months later without preceding symptoms [2]. This case raises awareness of mono- or oligoarticular arthritis as one of the signs of Lyme disease [1]. Prompt recognition of the disease leads to a faster diagnosis and better outcomes [6].

Research funding: None reported.

Author contributions: Both authors provided substantial contributions to conception and design, acquisition of data, or analysis and interpretation of data; both authors drafted the article or revised it critically for important intellectual content; both authors gave final approval of the version of the article to be published; and both authors agree to be accountable for all aspects of the work in ensuring that questions related to the accuracy or integrity of any part of the work are appropriately investigated and resolved.

Competing interests: None reported.

Informed consent: Verbal consent was obtained from the patient's mother. 


\section{References}

1. Miller JB, Aucott JN. Stages of Lyme arthritis. J Clin Rheumatol 2020. https://doi.org/10.1097/rhu.0000000000001513.

2. Schoen RT. Lyme disease: diagnosis and treatment. Curr Opin Rheumatol 2020;32:247-54.

3. Kugeler KJ, Schwartz AM, Delorey MJ, Mead PS, Hinckley AF. Estimating the frequency of Lyme disease diagnoses, United States, 2010-2018. Emerg Infect Dis 2021;27:616-9.

4. Schoen RT. Challenges in the diagnosis and treatment of Lyme disease. Curr Rheumatol Rep 2020;22:3.

5. Qureshi MZ, New D, Zulqarni NJ, Nachman S. Overdiagnosis and overtreatment of Lyme disease in children. Pediatr Infect Dis J 2002;21:12-4.

6. Saulsbury FT. Lyme arthritis in 20 children residing in a nonendemic area. Clin Pediatr 2005;44:419-21.

7. Lucente LN, Abu-Dayya A, Hennon T, Islam S, Wrotniak BH, Abdul Aziz R. Lyme arthritis in children on the uptick in western New York area. Clin Med Insights Arthritis Musculoskelet Disord 2019;12:117954411989085.

8. Madison-Antenucci S, Kramer LD, Gebhardt LL, Kauffman E. Emerging tick-borne diseases. Clin Microbiol Rev 2020;33: e00083-18.

9. Esposito S, Bosis S, Sabatini C, Tagliaferri L, Principi N. Borrelia burgdorferi infection and Lyme disease in children. Int J Infect Dis 2013;17:e153-8.
10. Nigrovic LE, Neville DN, Balamuth F, Bennett JE, Levas MN, Garro AC. A minority of children diagnosed with Lyme disease recall a preceding tick bite. Ticks Tick Borne Dis 2019;10: 694-6.

11. Nigrovic LE, Bennett JE, Balamuth F, Levas MN, Chenard RL, Maulden $A B$, et al. Accuracy of clinician suspicion of Lyme disease in the emergency department. Pediatrics 2017;140: e20171975.

12. Daikh BE, Emerson FE, Smith RP, Lucas FL, McCarthy CA. Lyme arthritis: a comparison of presentation, synovial fluid analysis, and treatment course in children and adults. Arthritis Care Res 2013;65:1986-90.

13. Aiyer A, Hennrikus W, Walrath J, Groh B, Ostrov B. Lyme arthritis of the pediatric lower extremity in the setting of polyarticular disease. J Child Orthop 2014;8:359-65.

14. Arvikar SL, Steere AC. Diagnosis and treatment of Lyme arthritis. Infect Dis Clin 2015;29:269-80.

15. Centers for Disease Control and Prevention. Lyme disease. Available from: https://www.cdc.gov/lyme/healthcare/ clinician_twotier.html [Accessed 31 May 2021].

16. Centers for Disease Control and Prevention. Lyme disease. Available from: https://www.cdc.gov/lyme/treatment/ LymeArthritis.html [Accessed 17 Aug 2021].

17. Volokitin M, Sheikh A, Patel S, Milani S, Banihashem M. Treating Bell's Palsy with osteopathic manipulative medicine: a case report. Cureus 2020. https://doi.org/10.7759/cureus. 11092. 